\title{
Early Steps in the DNA Base Excision Repair Pathway of a Fission Yeast Schizosaccharomyces pombe
}

\author{
Kyoichiro Kanamitsu and Shogo Ikeda \\ Department of Biochemistry, Faculty of Science, Okayama University of Science, 1-1 Ridai-cho, Kita-ku, Okayama 700-0005, Japan \\ Correspondence should be addressed to Shogo Ikeda, ikeda@dbc.ous.ac.jp
}

Received 21 June 2010; Accepted 12 August 2010

Academic Editor: Ashis Basu

Copyright ( $) 2010$ K. Kanamitsu and S. Ikeda. This is an open access article distributed under the Creative Commons Attribution License, which permits unrestricted use, distribution, and reproduction in any medium, provided the original work is properly cited.

\begin{abstract}
DNA base excision repair (BER) accounts for maintaining genomic integrity by removing damaged bases that are generated endogenously or induced by genotoxic agents. In this paper, we describe the roles of enzymes functioning in the early steps of BER in fission yeast. Although BER is an evolutionarily conserved process, some unique features of the yeast repair pathway were revealed by genetic and biochemical approaches. AP sites generated by monofunctional DNA glycosylases are incised mainly by AP lyase activity of Nth1p, a sole bifunctional glycosylase in yeast, to leave a blocked 3 ' end. The major AP endonuclease Apn2p functions predominantly in removing the $3^{\prime}$ block. Finally, a DNA polymerase fills the gap, and a DNA ligase seals the nick (Nthlpdependent or short patch BER). Apn1p backs up Apn2p. In long patch BER, Rad2p endonuclease removes flap DNA containing a lesion after DNA synthesis. A UV-specific endonuclease Uvelp engages in an alternative pathway by nicking DNA on the $5^{\prime}$ side of oxidative damage. Nucleotide excision repair and homologous recombination are involved in repair of BER intermediates including the AP site and single-strand break with the 3' block. Other enzymes working in 3' end processing are also discussed.
\end{abstract}

\section{Introduction}

DNA molecules in cells always suffer from chemical decay due to exposure to endogenous and environmental agents [1-3]. Cells die when the damage to DNA obstructs replication and transcription. Moreover, base damage causes mutations, which are responsible for cancer, aging, and the hereditary diseases [3-5]. Base excision repair (BER) is a DNA repair pathway directed mainly at nonbulky lesions, such as, alkylated and oxidized bases, and at some types of mismatched bases that are produced during replication or by deamination [3,5-7]. The BER pathway is usually initiated by DNA glycosylase that removes damaged bases to leave apurinic/apyrimidinic (AP) sites. The AP sites are further processed by an AP endonuclease that cleaves phosphodiether bonds $5^{\prime}$ to the AP site to leave a $3^{\prime} \mathrm{OH}$ and $5^{\prime}$ deoxyribose phosphate (5'-blocked end). Bifunctional DNA glycosylase associated with AP lyase removes damaged bases and cleaves $3^{\prime}$ to the AP site, leaving a $3^{\prime}-\alpha, \beta$-unsaturated aldehyde ( $3^{\prime}$-blocked end) and a $5^{\prime}$ phosphate. These blocked ends are subsequently converted into $3^{\prime} \mathrm{OH}$ and $5^{\prime}$ phosphate by appropriate enzymes. Finally, a repair DNA polymerase fills the gap, and a DNA ligase seals the nick.

The basic mechanism of BER was first elucidated in Escherichia coli [3]. Subsequent studies showed that the process is conserved in eukaryotes including the budding yeast Saccharomyces cerevisiae and mammals. Fission yeast (Schizosaccharomyces pombe) is an ascomycetous yeast that shares many fundamental cellular properties with higher multicellular eukaryotes [8]. Although $S$. pombe has been used as a prominent model organism, research of the BER pathway in yeast has started late. Completion of the S. pombe genome project in 2002 provided a list of the yeast BER machineries including DNA glycosylases and AP endonucleases, which are evolutionarily conserved from bacteria to man [9]. Over the past decade, the BER pathway of $S$. pombe has been fairly well characterized by genetic approaches using many BER-defective mutants constructed by gene targeting. Biochemical properties of BER enzymes were also examined using purified recombinant proteins. In this paper, we describe the roles of the enzymes involved in the early steps of BER in fission yeast with an emphasis on 
TABLE 1: DNA glycosylases in bacteria, yeasts, and human cells.

\begin{tabular}{|c|c|c|c|c|}
\hline E. coli & S. cerevisiae & S. pombe & Human & AP lyase \\
\hline Ung & Unglp & Unglp & hUNG & \\
\hline \multirow[t]{4}{*}{ Mug } & & Thplp & hTDG & \\
\hline & & & hSMUG1 & \\
\hline & & & hMBD4 & \\
\hline & & & & - \\
\hline \multirow[t]{2}{*}{ AlkA } & Mag1p & Maglp & & \\
\hline & & Mag2p & & \\
\hline \multicolumn{5}{|l|}{ TagA } \\
\hline & & & hMPG & \\
\hline MutY & & Myh1p & hMYH & \\
\hline \multirow[t]{3}{*}{ Nth1 } & Ntg1p & Nthlp & hNTH1 & \\
\hline & Ntg2p & & & \\
\hline & Ogglp & & hOGG1 & + \\
\hline \multicolumn{5}{|l|}{ Fpg } \\
\hline \multirow[t]{3}{*}{ Nei } & & & hNEIL1 & \\
\hline & & & hNEIL2 & \\
\hline & & & hNEIL3 & \\
\hline
\end{tabular}

outlining common features as well as differences with other model organisms.

\section{DNA Glycosylases in S. pombe}

DNA glycosylases identified in E. coli, S. cerevisiae, S. pombe, and the human are classified in Table 1 on the basis of their substrate specificities and structural features $[3,7,10]$. Monofunctional DNA glycosylase simply hydrolyzes the $N$ glycosidic bond to release damaged bases and leaves the AP site. Nth1 and Ogg1 DNA glycosylases are associated with AP lyase activity, which cleaves $3^{\prime}$ to the AP site by $\beta$ elimination, leaving a $3^{\prime}-\alpha, \beta$-unsaturated aldehyde. $E$. coli Fpg and Nei and human NEILs are other types of bifunctional glycosylases which cleave the AP site by $\beta, \delta$ elimination and generate a $3^{\prime}$-phosphate end. S. pombe has five monofunctional DNA glycosylases (Ung1p, Thp1p, Mag1p, Mag2p, and Myh1p) [11]. Unlike other organisms, Nth1p is a sole DNA glycosylase with AP lyase activity in $S$. pombe cells. Nth1 DNA glycosylase is $\beta$ lyase and does not possess $\delta$ lyase activity $[2,5,7]$.

\subsection{Uracil DNA Glycosylases Ung1p and Thp1p Function} in Avoidance of Spontaneous Mutation. Deamination of cytosine residues in DNA occurs spontaneously in $\mathrm{pH}$ - and temperature-dependent reactions, and results in conversion to uracil $[1,3]$. Cytosine deamination is estimated to produce 400 uracil residues per mammalian genome per day [12]. Promutagenic $U: G$ mispair causes $C: G$ to $T: A$ transition mutations, if not repaired prior to DNA replication.
Uracil also occurs in DNA through incorporation of dUMP instead of dTMP by DNA polymerase during replication. Deamination of 5-methylcytosine in DNA results in the formation of thymine, and hence $T: G$ mispairs. Uracil residues can be removed through the activity of uracil DNA glycosylase (UDG), which consists of four distinct families in mammalian cells: the UNG family (the major activity of UDG in cells), the MUG (mismatch-specific uracil DNA glycosylase)/TDG (thymine DNA glycosylase) family, the SMUG (single strand-specific monofunctional uracil-DNA glycosylase) family, and the MBD4 (methyl-binding domain 4) family $[3,5,13]$. The UDGs, other than MBD4, form a single protein superfamily with a common $\alpha / \beta$-fold structure and must have been evolved from a common ancestor [14].

$S$. pombe has two UDG genes, ung1 (systematic name SPCC1183.06) and thp1 (SPCC965.05c). The Ung1p protein is highly conserved ( $51 \%$ identity to human UNG) and localizes predominantly to the nucleus [15]. The bacterially expressed protein showed an apparent enzyme activity for uracil-containing DNA, which was inhibited by an inhibitor protein (UGI). Overexpression of ungl induces a DNA checkpoint-dependent cell cycle delay and causes cell death [15]. Human MUG/TDG was first isolated as an enzyme that excises thymine from $\mathrm{T}: \mathrm{G}$ mispairs as well as uracil from $\mathrm{U}: \mathrm{G}$ mispairs [16, 17]. S. pombe Thp1p is a member of the MUG family, and maintains a high level of glycosylase activity towards substrates containing U, 5-fluorouracil, 3,N $N^{4}$-ethenocytosine, and 5-hydroxyuracil [18]. Unlike human enzyme, Thp1p cannot excise thymine residues from the $T: G$ mispair substrate. Since cytosine methylation is undetectable in fission yeast cells, the potential to process this substrate appears to be correlated with the degree of cytosine methylation in the genome of organisms [19]. Thplp can also remove deaminated purine bases: xanthine and oxanine from guanine and hypoxanthine from adenine [20]. Both ungl and thpl mutants showed a moderate mutator phenotype [21]. Double mutation of the genes additively increased the mutation frequency. Moreover, expression of Unglp and Thplp suppressed spontaneous mutagenesis in UDG-deficient cells. These results indicate that both proteins play important roles in the prevention of spontaneous mutagenesis of $S$. pombe cells.

2.2. Two AlkA Homologs Mag1p and Mag2p Function in Removal of Alkylated Base. Alkylating agents such as methyl methanesulfonate (MMS) generate covalent modifications at nitrogen residues of DNA bases, in particular N3methyladenine (3-meA) and N7-methylguanine (7-meG), which account for $11 \%$ and $83 \%$ among the alkylation bases by MMS, respectively [1, 3]. In contrast, 7-meG appears to be a harmless alteration while 3-meA is a cytotoxic lesion that blocks both replication and transcription. $S$-adenosylmethionine, which is a common cosubstrate involved in methyl group transfer, also takes part in the nonenzymatic methylation of DNA [1]. In a human cell, 3-mA and 7-mG spontaneously generate 600 and 4,000 residues a day, respectively [12].

In E. coli, alkylation products such as 3-meA and 7meG are mainly removed by two structurally different 
monofunctional DNA glycosylases, AlkA and TagA [3]. The S. pombe genome encodes two alkA paralogs mag1 (SPAPB24D3.04c) and mag2 (SPBC23G7.11). The amino acid sequences of Mag1p and Mag2p share $44.8 \%$ similarity. The $S$. pombe mag1 gene was cloned by its ability to reverse the MMS-sensitive phenotype of an E. coli alkA/tagA double mutant [22]. The substrate range of Maglp overproduced in E. coli is limited to the main alkylation products, such as, 3-meA, 3-meG, and 7-meG, whereas no significant activity was found toward deamination products, ethenoadducts, or oxidation products [23]. The efficiency of 3-meA and 3-meG removal was 5-10 times slower for Mag1p than for E. coli AlkA, whereas the two enzymes remove 7-meG at a similar rate. On the other hand, biochemical analysis of Mag2p has not been performed yet because the recombinant protein expressed in E. coli showed no glycosylase activity [23].

Mutant strains mag $1 \Delta$ and mag $2 \Delta$ hardly showed MMS sensitivity [23-25]. The BER mutants $n t h 1 \Delta$, apn $2 \Delta$, and $\operatorname{rad} 2 \Delta$ were sensitive to MMS, while the double mutants $n$th $1 \Delta / \operatorname{mag} 1 \Delta$, apn $2 \Delta / \operatorname{mag} 1 \Delta$, and $\mathrm{rad} 2 \Delta / \operatorname{mag} 1 \Delta$ restored resistance to MMS $[23,25]$. This showed that Maglp is involved in the initial step of MMS-damaged repair both by the Nth1p-dependent short patch BER pathway and by the Rad2p (flap endonuclease)-dependent long patch BER pathway (Figure 1). Moreover, double mutant $n t h 1 \Delta / \operatorname{mag} 2 \Delta$ was more resistant to MMS than $n t h 1 \Delta$, indicating that Mag2p also functions in the removal of alkylated bases [25]. rad16 encodes an XPF homolog that functions as a DNA repair endonuclease in nucleotide excision repair (NER). The double mutant of rad16 with mag1 or mag2 increased sensitivity to MMS [25]. In addition, the $\operatorname{rad} 16 \Delta / \operatorname{mag} 1 \Delta / \operatorname{mag} 2 \Delta$ triple mutant exhibited the highest MMS sensitivity. Expression of Mag1p or Mag2p in the triple mutant restored tolerance to MMS. These results showed that BER and NER could independently repair the alkylation DNA damage. Comparison of the substrate specificity and kinetic parameters of Mag1p and Mag2p will be needed to dissect the precise roles of these redundant enzymes in MMS resistance. rhp51 encodes a RecA-like protein that functions in homologous recombination (HR). The double mutant of rhp51 with mag1 decreased the sensitivity to chronic MMS exposure compared with $\operatorname{rhp51\Delta }$ single mutant [24]. In addition, spontaneous intrachromosomal recombination frequencies increased 3-fold in the mag1 mutant [23]. These results show that both Maglp and HR contribute to repair of the alkylation base. Deletion of mag1 from $n t h 1$, rad16, or rad2 decreased the recombination frequency, indicating that AP sites generated by Maglp removal of spontaneous base lesions are substrates of short patch BER, long patch BER, NER, and HR [23].

2.3. Nth1p Is the Major Contributor for Incision of the AP Site. An 8-oxoguanine (8-oxoG) produced by the oxidation of the guanine residue in $\mathrm{G}$ : $\mathrm{C}$ could pair not only with cytosine but also with adenine, and the $\mathrm{G}$ : $\mathrm{C}$ to $\mathrm{T}$ : A mutation occurs during the next stage of replication $[3,5,7]$. In a human cell, about 1,000 residues of 8 -oxoG are generated per genome in a day [12]. Bifunctional DNA glycosylases Fpg (MutM) in $E$. coli and OGG1 in budding yeast and mammals can excise 8-oxoG paired with cytosine. Thymine glycol ( $\mathrm{Tg}$ ), which is the oxidation product of thymine, interrupts DNA replication and transcription. In human cells, production of $\mathrm{Tg}$ and similar oxidized pyrimidines is estimated to be 500 residues per genome per day [12]. In E. coli endonuclease III (Nth) and endonuclease VIII (Nei) excise Tg and oxidized pyrimidines from DNA and subsequently cleave the strand by AP lyase activity [3]. S. pombe has no homolog of Fpg, OGG1, or Nei [11].

The S. pombe nth1 gene (SPAC30D11.07) was cloned on the basis of homology to E. coli nth [26]. Nth1 family proteins have a helix-hairpin-helix motif in the vicinity of the active lysine residue and an iron-sulfur cluster [4Fe-4S] at the C terminal. In addition, a eukaryote's enzyme has a nuclear localization signal. Indeed, fusion protein of $S$. pombe Nth1p with green fluorescent protein was predominantly localized in the nucleus [27]. Unlike budding yeast Ntglp and the enzyme in mammals, $S$. pombe Nth1p was not observed in mitochondria.

A recombinant $S$. pombe Nth1p expressed in E. coli shows a broad substrate specificity. Mass spectrometry of released bases from oxidized DNA by treatment of Nth1p revealed that the enzyme efficiently excised five pyrimidine-derived lesions: 5-hydroxycytosine, Tg, 5hydroxy-6-hydrothymine, 5, 6-dihydroxycytosine, and 5hydroxyuracil [28]. S. pombe Nth1p could remove 5formyluracil and 5-hydroxymethyluracil as efficiently as $E$. coli Nth [29]. Moreover, 8-oxoG in 8-oxoG : G and 8-oxoG : a mispair is also the substrate for $S$. pombe Nth1p. The expression of $S$. pombe Nth1p reduced hydrogen peroxide $\left(\mathrm{H}_{2} \mathrm{O}_{2}\right)$ toxicity and the frequency of spontaneous mutations in the E. coli nth/nei double mutant [29]. Although the S. pombe $n t h 1 \Delta$ is sensitive to MMS, Nth1p did not remove 3-meA and 7-meG [30]. However, methyl-formamidepyrimidine, a cytotoxic lesion generated from $7-\mathrm{meG}$ by opening the imidazole ring, was excised efficiently by Nth1p. Nicking activity to oligonucleotides containing $\mathrm{Tg}$ and the $\mathrm{AP}$ site virtually disappeared in the extract from $n t h 1 \Delta$ cells, indicating that these lesions are mainly incised by Nth1p in the yeast cell [27].

An $n t h 1 \Delta$ strain was tolerant to oxidative damage $\left(\mathrm{H}_{2} \mathrm{O}_{2}\right.$ and menadione) and UV irradiation, but exhibited moderate sensitivity to MMS [27, 30, 31]. In addition, the mutant strain exhibited a more than 6-fold increase in the frequency of interchromosomal recombination [30]. Epistasis analysis of the nth1 gene versus rad2, rad16, and rhp55 showed that MMS damage could be repaired through NER and HR other than the BER pathway [30]. Although a mutant of the major AP endonuclease gene apn2 showed hypersensitivity to MMS, double mutation of $n$ th1 and apn2 became tolerant to MMS [31]. This shows that Nth1p functions upstream of Apn $2 p$ in the same pathway; that is, the $3^{\prime}$-blocked end generated by Nth1p is converted to $3^{\prime} \mathrm{OH}$ by phosphodiesterase activity of Apn2p (Figure 1). In $S$. pombe cells, Nth1p not only removes the oxidized base as DNA glycosylase, but also incises a large portion of the AP site generated by the action of Mag1p and Mag2p DNA glycosylases. Thus, Nth1p plays a central role during early steps of the BER pathway in the fission yeast [27]. 


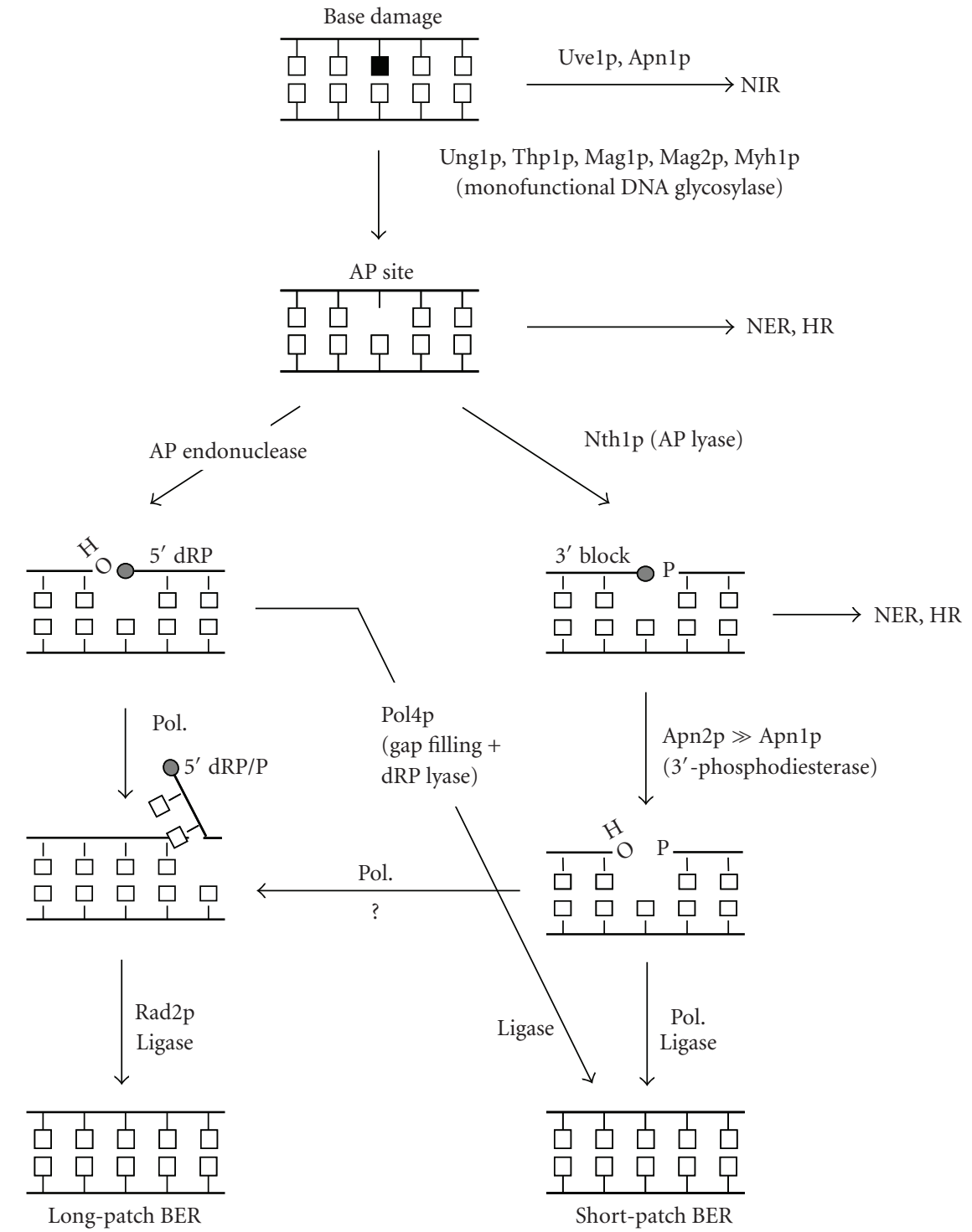

FIGURE 1: A schematic illustration of the BER pathway in S. pombe. In the Nthlp-dependent (or short patch) pathway, repair of the AP site is initiated by incision of Nth1p leaving the $3^{\prime}$-block. Apn2p functions primarily in the removal of the $3^{\prime}$-block, and poorly in the incision of AP sites. Apn1p is a back-up enzyme for $3^{\prime}$-phosphodiesterase activity of Apn2p. Finally, a DNA polymerase (Pol.) fills the gap, and a DNA ligase seals the nick. NER and HR could repair a part of the AP sites and single-strand breaks with $3^{\prime}$ blocks. AP endonuclease incises the AP site leaving $5^{\prime}$-dRP end, which will be removed by $5^{\prime}$-dRP lyase activity of Pol4p to feed into short-patch BER. In the long patch pathway, Rad2p incises the flap DNA containing the lesion after DNA synthesis. Uvelp and Apn1p are possibly involved in the NIR pathway. Details are discussed in the text.

2.4. DNA Glycosylase for Mismatch. An 8-oxoG: A mispair in DNA, which is generated by incorporation of an adenine residue opposite to 8 -oxoG, leads to a $\mathrm{G}$ : $\mathrm{C}$ to $\mathrm{T}$ : A mutation $[3,5]$. The repair of this mismatch is initiated by excision of an adenine residue by adenine-specific mismatch DNA glycosylase (MutY in E. coli). The mutY homolog of $S$. pombe, namely, myh1 (SPAC26A3.02), has been cloned [32]. Expression of Myhlp in the mutY mutant of E. coli could reduce the spontaneous mutation frequency of the cells. Purified Myh1p recognized A:G and A:8-oxoG, as well as 2-aminopurine: $\mathrm{G}$ and $\mathrm{A}: 2$-aminopurine [32]. In addition,
Myhlp probably prevents a $\mathrm{C}: \mathrm{G}$ to $\mathrm{G}: \mathrm{C}$ transversion mutation because it can remove the guanine from $\mathrm{G}: 8$ oxoG [33]. The $m y h 1 \Delta$ strain displays a 36-times higher frequency of spontaneous mutation than the wild strain [34]. Moreover, $m y h 1 \Delta$ showed sensitivity to $\mathrm{H}_{2} \mathrm{O}_{2}$. Myhlp binds to proliferative cell nuclear antigen (PCNA) and PCNA-like heterotrimer Rad9p/Rad1p/Hus1p [35-37]. All subunits of the latter complex are required for proper functioning of the checkpoint and DNA replication under stress [38]. Myh1p may act as an adaptor to recruit checkpoint proteins to the DNA lesion [36]. 
TABLE 2: AP endonucleases in bacteria, yeasts, and human cells.

\begin{tabular}{lccc}
\hline E. coli & S. cerevisiae & S. pombe & Human \\
\hline Xth & Apn2p & Apn2p & hAPE1 \\
& Apn1p & $\begin{array}{c}\text { Apn1p } \\
\text { (Uve1p) }\end{array}$ & \\
\hline Nfo & Apn &
\end{tabular}

AP endonucleases showed in boldface contribute to the major activities in each organism.

\section{AP Endonucleases in S. pombe}

AP sites are generated by removal of damaged bases by monofunctional DNA glycosylase and more frequently by nonenzymatic hydrolysis of the $N$-glycosidic bond of DNA $[1,3]$. In a human cell, about 9,000 residues of the AP sites arise per genome in a day [12]. AP endonuclease hydrolyzes phosphodiether bonds $5^{\prime}$ to the AP site to leave $3^{\prime} \mathrm{OH}$ and $5^{\prime}$-deoxyribose phosphate (dRP). E. coli has two types of AP endonuclease, exonuclease III (Xth), and endonuclease IV ( $\mathrm{Nfo})$ [3, 39]. AP endonucleases from various organisms are classified on the basis of structural similarity to Xth and Nfo (Table 2). APE1 in mammals is an Xth-type homolog, which accounts for more than $90 \%$ of the AP endonuclease activity in cells $[3,39]$. A second Xth-type homolog APE2 was found [40, 41], but no Nfo-type enzymes were present in mammalian cells. On the other hand, yeasts have an Nfo-type homolog Apn1p other than Apn2p with similarity to mammalian APE2. In the budding yeast, Apn1p is the major contributor of AP endonuclease activity [39]. Both Xth and Nfo types of AP endonuclease could also convert $3^{\prime}$ blocked ends (e.g., $3^{\prime}$ $\alpha, \beta$-unsaturated aldehyde, phosphoglycolate, and phosphate termini) to $3^{\prime} \mathrm{OH}$. Therefore, $3^{\prime}$-phosphodiesterase and $3^{\prime}$ phosphatase activity of AP endonuclease is essential for the repair of single-strand breaks induced by reactive oxygen species (ROS), ionized radiation, and bleomycin $[39,42]$.

\subsection{Apn2p Predominantly Functions in the Removal of $3^{\prime}$} Blocks. The S. pombe apn2 gene (SPBC3D6.10) encodes an AP endonuclease with homology to $S$. cerevisiae Apn2p and human APE2 [40, 41, 43, 44]. The N-terminal region of the protein has homology with Xth. Notably, Apn2p maintains the entire critical catalytic residue of hAPE1, which is also conserved in several proteins recently predicted to maintain the four-layered $\alpha / \beta$ of Xth and the "nonspecific" nuclease DNase I $[45,46]$. The C-terminal region of Apn2p protein contains a binding motif similar to PCNA and a sequence homologous to DNA topoisomerase III (TOP3) family [40, 41, 47]. Physical interaction of S. cerevisiae Apn2p with PCNA stimulates the $3^{\prime}$ to $5^{\prime}$ exonuclease and $3^{\prime}$ phosphodiesterase activities of Apn2p in vitro [47]. However, the PCNA-binding motif and the TOP3-homologous region of $S$. pombe Apn2p are dispensable for BER of MMS damage in cells $[44,48]$. It remains to be elucidated whether Apn2p binds to $S$. pombe PCNA in vitro and in vivo. Apn $2 \mathrm{p}$ is predominantly localized in the nucleus [49].
Cells lacking Apn2p exhibit severe hypersensitivity to treatment with MMS and bleomycin analogues [31, 44, 49]. The mutation rate of $a p n 2 \Delta$ cells rose remarkably by MMS [44]. Incision activity to oligonucleotides containing an AP site (or tertahydrofuran) was significantly reduced in the extract from apn $2 \Delta$ cells $[44,49]$. These results show that Apn2p is the major contributor of AP endonuclease activity in S. pombe cells. Disruption of $n$th 1 in apn $2 \Delta$ cells restored tolerance to MMS, indicating that Apn2p functions downstream of Nth1p in the same pathway (Figure 1). Although overexpression of Apn2p in $n t h 1 \Delta$ failed to suppress MMS sensitivity, hAPE1 and S. cerevisiae Apn1p reduced the MMS sensitivity of $n t h 1 \Delta$ cells to the wild type level [27, 49]. This result suggests that Apn2p cannot begin repair of the AP site in cells in spite of the fact that the enzyme can cleave the AP site in vitro. S. pombe Apn2p predominantly functions in the removal of $3^{\prime}$ blocks induced by Nthlp. Trans-complementation of $S$. cerevisiae Apnlp strongly reflects differences in the major pathway for processing the AP sites between S. cerevisiae and S. pombe cells.

\subsection{Other AP Endonucleases of S.pombe. apn1} (SPCC622.17) encodes an Nfo-type AP endonuclease [50]. Although the APN1 mutant of S. cerevisiae is hypersensitive to MMS, the $S$. pombe apn1 mutant exhibited no sensitivity to MMS and oxidative stress. In addition, the $n t h 1 \Delta / a p n 1 \Delta$ double mutant showed the same MMS sensitivity as the

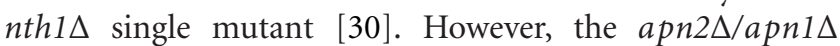
double mutant was more sensitive than the apn2 $\Delta$ single mutant [44]. Moreover, when Apn1p was overexpressed in apn2 2 cells, MMS sensitivity was partially restored [49]. Therefore, Apn1p removes the 3 -blocked end as a back-up function of the major enzyme Apn2p (Figure 1). Since Apn1p was observed in both the nucleus and cytoplasm, it possibly functions in more than just nuclear DNA repair [49]. S. cerevisiae Apn1p has been shown to move into the mitochondria to maintain genomic stability [51].

Uvelp (SPBC19C7.09c), which is primarily a UVphotoproduct specific endonuclease of $S$. pombe, could recognize non-UV-induced DNA damage (e.g., the AP site and dihydrouracil) and mismatched bases in vitro, and hydrolyze immediately $5^{\prime}$ to the damage $[52,53]$. The protein has a TIM barrel fold that is very similar to the structure of Nfo [54]. S. cerevisiae has no reported Uvelp homolog. Deletion of the uve1 gene in apn2s significantly increased MMS sensitivity [44]. Neurospora crassa Uvelp could complement E. coli $x \operatorname{th} \Delta / n$ fo $\Delta$ with respect to sensitivity to MMS and $t$ butyl hydroperoxide [55]. The mutation frequency of uve1 $\Delta$ increased when treated with $\mathrm{H}_{2} \mathrm{O}_{2}$ [56]. This effect was additively elevated by the deletion of apn2, indicating that both Uve1p and Apn2p contribute to the avoidance of $\mathrm{H}_{2} \mathrm{O}_{2}$ induced mutagenesis, but these enzymes define a distinct oxidative damage repair pathway. E. coli $\mathrm{Nfo}$ and S. cerevisiae Apnlp can nick DNA on the $5^{\prime}$ side of several oxidized base lesions, generating a $3^{\prime} \mathrm{OH}$ and a $5^{\prime}$ dangling damaged nucleotide, which provide an alternative nucleotide incision repair (NIR) pathway to the classic BER $[57,58]$. Therefore, S. pombe Uvelp and Apnlp possibly engage in the NIR 
pathway by nicking oxidative damage on DNA in an Nthlpindependent manner (Figure 1). In such a case, damaged bases might be removed by a flap endonuclease, $\operatorname{Rad} 2 p$, after long-patch repair DNA synthesis is primed by the actions of Uvelp and Apn1p [59,60]. Uvelp works efficiently in mitochondria as well as in the nucleus [61].

3.3. Short- and Long-Patch Repair Subpathways. In the Nth1dependent repair pathway, 3'-phosphodiesterase activity of AP endonuclease removes $3^{\prime}$-blocked end resulting in a 1nt gap, which will be refilled via short-patch BER (Figure 1). However, it is not known whether the BER synthesis extends beyond a single nucleotide, with incorporation of two or more nucleotides (long-patch BER). When AP endonuclease incises the AP site, single-strand break with $3^{\prime}-\mathrm{OH}$ and $5^{\prime}-\mathrm{dRP}$ ends is generated. The dRP moiety is released by combined action of a DNA polymerase and Rad2p endonuclease (long-patch BER). An X-family DNA polymerase of $S$. pombe, Pol4p, has been shown to contain an intrinsic $5^{\prime}$ dRP lyase activity like mammalian DNA polymerases $\beta$ and $\lambda$, suggesting another AP endonuclease pathway to feed into short-patch BER (Figure 1) [62]. S. cerevisiae Trf4 protein plays a similar role in short-patch BER of the yeast [63]. In mammalian cells, the choice of short-patch or long-patch BER may depend on the state of the $5^{\prime}$ terminal moiety and protein-protein interaction of BER components [64]. In vitro analyses of repair synthesis with whole cell extracts or purified enzymes are needed to clarify the relative contribution of short-patch and long-patch BER in S. pombe cells.

\section{Enzymes for Removing $3^{\prime}$ Blocked Ends in S. pombe Cells}

Single-strand breaks with $3^{\prime}$ phosphate ends in DNA are induced by chemical attacks of endogenous ROS and environmental oxidants [3]. Fpg/Nei-type DNA glycosylases cleave the AP site by $\beta, \delta$ elimination and generate DNA strand breaks with a $3^{\prime}$-phosphate end. In human cells, removal of $3^{\prime}$ phosphate is dependent on $3^{\prime}$-DNA phosphatase (hPNK), and not the major AP endonuclease hAPE1 [65]. Unlike bacteria and humans, S. pombe does not possess Fpg/Nei-type DNA glycosylase, and the physiological source of $3^{\prime}$ phosphate in the yeast is probably due to the action of tyrosyl-DNA phosphodiesterase I (TDP1). TDP1 catalyzes the hydrolysis of 3 '-phosphotyrosyl bonds of the irreversible topoisomerase I (Top1)-DNA covalent complex to generate a $3^{\prime}$-phosphate end $[66,67]$. A $3^{\prime}$-DNA phosphatase (Pnk1p; SPAC23C11.04c) has been purified from $S$. pombe on the basis of its ability to process $\mathrm{H}_{2} \mathrm{O}_{2}$ damaged DNA to allow DNA synthesis by DNA polymerase [68]. Like human PNK, Pnk1p localizes in nuclei and has both $5^{\prime}$-DNA kinase and $3^{\prime}$-DNA phosphatase activities [69]. The pnk1 mutant showed hypersensitivity to ionizing radiation and camptothecin, an inhibitor Top1, but not to MMS. It remains to be elucidated whether Pnk1p is the major 3 '-DNA phosphatase in $S$. pombe cells.

TDP1 has been implicated in the repair of the irreversible Top1-DNA covalent complex, which can be generated by either exogenous or endogenous factors [67]. Additionally, TDP1 hydrolyses a variety of $3^{\prime}$ lesions, including $3^{\prime}$ phosphoglycolate $[70,71]$. A recessive mutation in the human (TDP1) gene is responsible for the inherited disorder, spinocerebellar ataxia with axonal neuropathy (SCAN1) [72]. A $t d p 1$ (SPCP31B10.05) mutant of S. pombe was completely sensitive to camptothecin [73]. Moreover, the mutant cells progressively accumulate DNA damage and rapidly lose viability in nondividing conditions [73]. Low cellular respiration levels protected the $t d p 1 \Delta$ cells, indicating that the production of endogenous ROS is a major cause for the accumulation of DNA lesions in the absence of Tdp1p [73]. Therefore, Tdplp with Pnk1p processes the same naturally occurring $3^{\prime}$ ends, produced from oxidative DNA damage. Rapid and extensive death of the $t d p 1 \Delta / r h p 51 \Delta$ double mutant strain suggested the pivotal role of the HR process in DNA repair in $t d p 1 \Delta$ cells [73].

\section{BER in a Catalase-Deficient Mutant of $S$. pombe}

The BER mutants of $S$. pombe are substantially resistant to $\mathrm{H}_{2} \mathrm{O}_{2}$. A catalase-deficient mutant $(c t t 1 \Delta)$, in which ROS scavenging activity is extensively reduced, became sensitive to $\mathrm{H}_{2} \mathrm{O}_{2}[74,75]$. Deletion of the BER gene (nth1, apn1, apn2, or uve1) from ctt $1 \Delta$ further increased the sensitivity to $\mathrm{H}_{2} \mathrm{O}_{2}$, indicating that catalase activity obscures the functions of BER enzymes in vivo [75]. Double mutants in both $c t t 1$ and BER genes showed extremely high spontaneous mutation rates, especially in the $c t t 1 / n t h 1$ mutant. Vitamin $C$ relieved the mutator phenotype of the ctt1/nth1 mutant [75]. The results provide evidence that BER enzymes as well as catalase and an antioxidant contribute in vivo to avoidance of ROSinduced mutagenesis and cell death.

\section{Conclusion}

In this paper, we described the early steps of BER in the fission yeast highlighting the key roles of DNA glycosylases, AP endonucleases, and other end-cleaning activities in maintenance of genomic integrity. Although the yeast BER pathway consists of evolutionarily conserved enzymes, the major activity for processing the AP site is different from these of the budding yeast and mammals. AP sites generated by monofunctional glycosylase are mainly repaired via the Nth1p-dependent BER pathway [23, 27, 30, 31]. Unlike other model organisms, the major AP endonuclease of this yeast is assigned to Apn2p, which works primarily in the removal of the $3^{\prime}$ block, and poorly in the incision of AP sites [23, $44,49]$. Uvelp engages in an Nthlp-independent pathway by nicking DNA on the $5^{\prime}$ side of oxidative damage $[56,75]$. Genetic interactions of BER genes with NER and HR genes suggest synergism among the different DNA repair pathways in the protection of alkylation and oxidative damage [23-25, 30]. NER and HR probably repair the intermediates of BER such as, the AP site and single-strand breaks with $3^{\prime}$ blocks. BER operates most efficiently when specific protein-protein coordination occurs [76]. A study of physical interaction 
of the BER proteins will facilitate an understanding of the regulation of BER protein activities and crosstalk between BER and other DNA transaction pathways in yeast cells. Upon nitrogen-starvation, mostly $\mathrm{G}_{2}$ vegetative $S$. pombe cells promote two rounds of division and enter the $G_{0}$ state with 1C DNA [77]. The S. pombe $\mathrm{G}_{0}$ state will provide an excellent model to reconsider the roles of BER and other DNA pathways in resting and nondividing physiological conditions $[73,78]$.

\section{Acknowledgment}

This work was supported in part by the Ministry of Education, Culture, Sports, Science and Technology of Japan through a Financial Assistance Program of the Social Cooperation Study (2006-2010).

\section{References}

[1] T. Lindahl, "Instability and decay of the primary structure of DNA," Nature, vol. 362, no. 6422, pp. 709-715, 1993.

[2] R. De Bont and N. van Larebeke, "Endogenous DNA damage in humans: a review of quantitative data," Mutagenesis, vol. 19, no. 3, pp. 169-185, 2004.

[3] E. C. Friedberg, G. C. Walker, W. Siede, R. D. Wood, R. A. Schultz, and T. Ellenberger, DNA Repair and Mutagenesis, ASM Press, Washington, DC, USA, 2nd edition, 2006.

[4] M. S. Cooke, M. D. Evans, M. Dizdaroglu, and J. Lunec, "Oxidative DNA damage: mechanisms, mutation, and disease," FASEB Journal, vol. 17, no. 10, pp. 1195-1214, 2003.

[5] D. E. Barnes and T. Lindahl, "Repair and genetic consequences of endogenous DNA base damage in mammalian cells," Annual Review of Genetics, vol. 38, pp. 445-476, 2004.

[6] H. Nilsen and H. E. Krokan, "Base excision repair in a network of defence and tolerance," Carcinogenesis, vol. 22, no. 7, pp. 987-998, 2001.

[7] M. L. Hegde, T. K. Hazra, and S. Mitra, "Early steps in the DNA base excision/single-strand interruption repair pathway in mammalian cells," Cell Research, vol. 18, no. 1, pp. 27-47, 2008.

[8] M. Yanagida, "The model unicellular eukaryote, Schizosaccharomyces pombe," Genome Biology, vol. 3, no. 3, pp. 2003.12003.4, 2002.

[9] V. Wood, R. Gwilliam, M.-A. Rajandream et al., "The genome sequence of Schizosaccharomyces pombe," Nature, vol. 415, no. 6874, pp. 871-880, 2002.

[10] K. Hitomi, S. Iwai, and J. A. Tainer, "The intricate structural chemistry of base excision repair machinery: implications for DNA damage recognition, removal, and repair," DNA Repair, vol. 6, no. 4, pp. 410-428, 2007.

[11] O. Fleck, "DNA repair pathway," in The Molecular Biology of Schizosaccharomyces pombe, R. Egel, Ed., pp. 101-115, Springer, Berlin, Germany, 2004.

[12] T. A. Kunkel, "The high cost of living," Trends in Genetics, vol. 15, no. 3, pp. 93-94, 1999.

[13] S.-I. Yonekura, N. Nakamura, S. Yonei, and Q.-M. ZhangAkiyama, "Generation, biological consequences and repair mechanisms of cytosine deamination in DNA," Journal of Radiation Research, vol. 50, no. 1, pp. 19-26, 2009.
[14] L. Aravind and E. V. Koonin, "The alpha/beta fold uracil DNA glycosylases: a common origin with diverse fates," Genome Biology, vol. 1, no. 4, pp. research0007.1-research0007.87, 2000.

[15] R. T. Elder, X. Zhu, S. Priet et al., "A fission yeast homologue of the human uracil-DNA-glycosylase and their roles in causing DNA damage after overexpression," Biochemical and Biophysical Research Communications, vol. 306, no. 3, pp. 693-700, 2003.

[16] K. Wiebauer and J. Jiricny, "In vitro correction of G.T mispairs to G.C pairs in nuclear extracts from human cells," Nature, vol. 339, no. 6221, pp. 234-236, 1989.

[17] P. Neddermann, P. Gallinari, T. Lettieri et al., "Cloning and expression of human G/T mismatch-specific thymine-DNA glycosylase," The Journal of Biological Chemistry, vol. 271, no. 22, pp. 12767-12774, 1996.

[18] U. Hardeland, M. Bentele, J. Jiricny, and P. Schär, "The versatile thymine DNA-glycosylase: a comparative characterization of the human, Drosophila and fission yeast orthologs," Nucleic Acids Research, vol. 31, no. 9, pp. 2261-2271, 2003.

[19] D. Cortázar, C. Kunz, Y. Saito, R. Steinacher, and P. Schär, "The enigmatic thymine DNA glycosylase," DNA Repair, vol. 6, no. 4, pp. 489-504, 2007.

[20] L. Dong, R. Mi, R. A. Glass, J. N. Barry, and W. Cao, "Repair of deaminated base damage by Schizosaccharomyces pombe thymine DNA glycosylase," DNA Repair, vol. 7, no. 12, pp. 1962-1972, 2008.

[21] M. Ikeda, R. Ikeda, and S. Ikeda, "Spontaneous mutation in uracil DNA glycosylase-deficient cells of a fission yeast Schizosaccharomyces pombe," Current Topics in Biochemical Research, vol. 11, no. 1, pp. 55-60, 2009.

[22] A. Memisoglu and L. Samson, "Cloning and characterization of a cDNA encoding a 3-methyladenine DNA glycosylase from the fission yeast Schizosaccharomyces pombe," Gene, vol. 177, no. 1-2, pp. 229-235, 1996.

[23] I. Alseth, F. Osman, H. Korvald et al., "Biochemical characterization and DNA repair pathway interactions of Mag1mediated base excision repair in Schizosaccharomyces pombe," Nucleic Acids Research, vol. 33, no. 3, pp. 1123-1131, 2005.

[24] A. Memisoglu and L. Samson, "Contribution of base excision repair, nucleotide excision repair, and DNA recombination to alkylation resistance of the fission yeast Schizosaccharomyces pombe," Journal of Bacteriology, vol. 182, no. 8, pp. 2104-2112, 2000.

[25] K. Kanamitsu, H. Tanihigashi, Y. Tanita, S. Inatani, and S. Ikeda, "Involvement of 3-methyladenine DNA glycosylases Mag1p and Mag2p in base excision repair of methyl methanesulfonate-damaged DNA in the fission yeast Schizosaccharomyces pombe," Genes and Genetic Systems, vol. 82, no. 6, pp. 489-494, 2007.

[26] T. Roldán-Arjona, C. Anselmino, and T. Lindahl, "Molecular cloning and functional analysis of a Schizosaccharomyces pombe homologue of Escherichia coli endonuclease III," Nucleic Acids Research, vol. 24, no. 17, pp. 3307-3312, 1996.

[27] T. Sugimoto, E. Igawa, H. Tanihigashi, M. Matsubara, H. Ide, and S. Ikeda, "Roles of base excision repair enzymes Nthlp and Apn $2 \mathrm{p}$ from Schizosaccharomyces pombe in processing alkylation and oxidative DNA damage," DNA Repair, vol. 4, no. 11, pp. 1270-1280, 2005.

[28] B. Karahalil, T. Roldán-Arjona, and M. Dizdaroglu, "Substrate specificity of Schizosaccharomyces pombe Nth protein for products of oxidative DNA damage," Biochemistry, vol. 37, no. 2, pp. 590-595, 1998. 
[29] S.-I. Yonekura, N. Nakamura, T. Doi et al., "Recombinant Schizosaccharomyces pombe Nth1 protein exhibits DNA glycosylase activities for 8-oxo-7,8-dihydroguanine and thymine residues oxidized in the methyl group," Journal of Radiation Research, vol. 48, no. 5, pp. 417-424, 2007.

[30] F. Osman, M. Bjørås, I. Alseth et al., "A new Schizosaccharomyces pombe base excision repair mutant, nth1, reveals overlapping pathways for repair of DNA base damage," Molecular Microbiology, vol. 48, no. 2, pp. 465-480, 2003.

[31] I. Alseth, H. Korvald, F. Osman, E. Seeberg, and M. Bjørås, "A general role of the DNA glycosylase Nth1 in the abasic sites cleavage step of base excision repair in Schizosaccharomyces pombe," Nucleic Acids Research, vol. 32, no. 17, pp. 5119-5125, 2004.

[32] A.-L. Lu and W. P. Fawcett, "Characterization of the recombinant MutY homolog, an adenine DNA glycosylase, from yeast Schizosaccharomyces pombe," The Journal of Biological Chemistry, vol. 273, no. 39, pp. 25098-25105, 1998.

[33] T. Doi, S.-I. Yonekura, K. Tano, S. Yasuhira, S. Yonei, and Q.-M. Zhang, "The Shizosaccharomyces pombe homolog (SpMYH) of the Escherichia coli MutY is required for removal of guanine from 8-oxoguanine/guanine mispairs to prevent G:C to C:G transversions," Journal of Radiation Research, vol. 46, no. 2, pp. 205-214, 2005.

[34] D.-Y. Chang, Y. Gu, and A.-L. Lu, "Fission yeast (Schizosaccharomyces pombe) cells defective in the mutYhomologous glycosylase activity have a mutator phenotype and are sensitive to hydrogen peroxide," Molecular Genetics and Genomics, vol. 266, no. 2, pp. 336-342, 2001.

[35] D.-Y. Chang and A.-L. Lu, "Functional interaction of MutY homolog with proliferating cell nuclear antigen in fission yeast, Schizosaccharomyces pombe," The Journal of Biological Chemistry, vol. 277, no. 14, pp. 11853-11858, 2002.

[36] D.-Y. Chang and A.-L. Lu, "Interaction of checkpoint proteins Hus1/Rad1/Rad9 with DNA base excision repair enzyme MutY homolog in fission yeast, Schizosaccharomyces pombe," The Journal of Biological Chemistry, vol. 280, no. 1, pp. 408-417, 2005.

[37] K. Jansson, J. Warringer, A. Farewell et al., "The tumor suppressor homolog in fission yeast, $m y h 1^{+}$, displays a strong interaction with the checkpoint gene rad $1^{+}$," Mutation Research, vol. 644, no. 1-2, pp. 48-55, 2008.

[38] T. Caspari, M. Dahlen, G. Kanter-Smoler et al., "Characterization of Schizosaccharomyces pombe Hus1: a PCNA-related protein that associates with Rad1 Rad9," Molecular and Cellular Biology, vol. 20, no. 4, pp. 1254-1262, 2000.

[39] B. Demple and L. Harrison, "Repair of oxidative damage to DNA: enzymology and biology," Annual Review of Biochemistry, vol. 63, pp. 915-948, 1994.

[40] M. Z. Hadi and D. M. Wilson III, "Second human protein with homology to the Escherichia coli abasic endonuclease exonuclease III," Environmental and Molecular Mutagenesis, vol. 36, no. 4, pp. 312-324, 2000.

[41] D. Tsuchimoto, Y. Sakai, K. Sakumi et al., "Human APE2 protein is mostly localized in the nuclei and to some extent in the mitochondria, while nuclear APE2 is partly associated with proliferating cell nuclear antigen," Nucleic Acids Research, vol. 29, no. 11, pp. 2349-2360, 2001.

[42] J. L. Parsons, I. I. Dianova, and G. L. Dianov, "APE1 is the major 3 '-phosphoglycolate activity in human cell extracts," Nucleic Acids Research, vol. 32, no. 12, pp. 3531-3536, 2004.
[43] R. E. Johnson, C. A. Torres-Ramos, T. Izumi, S. Mitra, S. Prakash, and L. Prakash, "Identification of APN2, the Saccharomyces cerevisiae homolog of the major human AP endonuclease HAP1, and its role in the repair of abasic sites," Genes and Development, vol. 12, no. 19, pp. 3137-3143, 1998.

[44] B. Ribar, T. Izumi, and S. Mitra, "The major role of human AP-endonuclease homolog Apn2 in repair of abasic sites in Schizosaccharomyces pombe," Nucleic Acids Research, vol. 32, no. 1, pp. 115-126, 2004.

[45] M. Dlakić, "Functionally unrelated signalling proteins contain a fold similar to $\mathrm{Mg}^{2+}$-dependent endonucleases," Trends in Biochemical Sciences, vol. 25, no. 6, pp. 272-273, 2000.

[46] M. Z. Hadi, K. Ginalski, L. H. Nguyen, and D. M. Wilson III, "Determinants in nuclease specificity of Ape1 and Ape2, human homologues of Escherichia coli exonuclease III," Journal of Molecular Biology, vol. 316, no. 3, pp. 853-866, 2002.

[47] I. Unk, L. Haracska, X. V. Gomes, P. M. J. Burgers, L. Prakash, and S. Prakash, "Stimulation of $3{ }^{\prime}->5^{\prime}$ exonuclease and $3^{\prime}$-phosphodiesterase activities of yeast Apn2 by proliferating cell nuclear antigen," Molecular and Cellular Biology, vol. 22, no. 18, pp. 6480-6486, 2002.

[48] E. Igawa, H. Tanihigashi, A. Yamada, and S. Ikeda, "Functions of conserved motifs in the major AP endonuclease Apn2p of Schizosaccharomyces pombe for base excision repair of alkylation DNA damage," Current Topics in Biochemical Research, vol. 10, no. 1, pp. 101-105, 2008.

[49] H. Tanihigashi, A. Yamada, E. Igawa, and S. Ikeda, "The role of Schizosaccharomyces pombe DNA repair enzymes Apn1p and Uvelp in the base excision repair of apurinic/apyrimidinic sites," Biochemical and Biophysical Research Communications, vol. 347, no. 4, pp. 889-894, 2006.

[50] D. Ramotar, J. Vadnais, J.-Y. Masson, and S. Tremblay, "Schizosaccharomyces pombe Apn1 encodes a homologue of the Escherichia coli endonuclease IV family of DNA repair proteins," Biochimica et Biophysica Acta, vol. 1396, no. 1, pp. 15-20, 1998.

[51] R. Vongsamphanh, P.-K. Fortier, and D. Ramotar, "Pirlp mediates translocation of the yeast Apn $1 p$ endonuclease into the mitochondria to maintain genomic stability," Molecular and Cellular Biology, vol. 21, no. 5, pp. 1647-1655, 2001.

[52] A. M. Avery, B. Kaur, J.-S. Taylor, J. A. Mello, J. M. Essigmann, and P. W. Doetsch, "Substrate specificity of ultraviolet DNA endonuclease (UVDE/Uvelp) from Schizosaccharomyces pombe," Nucleic Acids Research, vol. 27, no. 11, pp. 2256-2264, 1999.

[53] B. Kaur, J. L. A. Fraser, G. A. Freyer, S. Davey, and P. W. Doetsch, "A Uvelp-mediated mismatch repair pathway in Schizosaccharomyces pombe," Molecular and Cellular Biology, vol. 19, no. 7, pp. 4703-4710, 1999.

[54] N. Goosen and G. F. Moolenaar, "Repair of UV damage in bacteria," DNA Repair, vol. 7, no. 3, pp. 353-379, 2008.

[55] S.-I. Kanno, S. Iwai, M. Takao, and A. Yasui, "Repair of apurinic/apyrimidinic sites by UV damage endonuclease; a repair protein for UV and oxidative damage," Nucleic Acids Research, vol. 27, no. 15, pp. 3096-3103, 1999.

[56] J. L. A. Fraser, E. Neill, and S. Davey, "Fission yeast Uve1 and Apn2 function in distinct oxidative damage repair pathways in vivo," DNA Repair, vol. 2, no. 11, pp. 1253-1267, 2003.

[57] A. A. Ischenko and M. K. Saparbaev, "Alternative nucleotide incision repair pathway for oxidative DNA damage," Nature, vol. 415, no. 6868, pp. 183-187, 2002. 
[58] A. A. Ishchenko, G. Sanz, C. V. Privezentzev, A. V. Maksimenko, and M. Saparbaev, "Characterisation of new substrate specificities of Escherichia coli and Saccharomyces cerevisiae AP endonucleases," Nucleic Acids Research, vol. 31, no. 21, pp. 6344-6353, 2003.

[59] R. Yonemasu, S. J. McCready, J. M. Murray et al., "Characterization of the alternative excision repair pathway of UV-damaged DNA in Schizosaccharomyces pombe," Nucleic Acids Research, vol. 25, no. 8, pp. 1553-1558, 1997.

[60] J. L. Alleva, S. Zuo, J. Hurwitz, and P. W. Doetsch, "In vitro reconstitution of the Schizosaccharomyces pombe alternative excision repair pathway," Biochemistry, vol. 39, no. 10, pp. 2659-2666, 2000.

[61] S. Yasuhira and A. Yasui, "Alternative excision repair pathway of UV-damaged DNA in Schizosaccharomyces pombe operates both in nucleus and in mitochondria," The Journal of Biological Chemistry, vol. 275, no. 16, pp. 11824-11828, 2000.

[62] S. González-Barrera, A. Sánchez, J. F. Ruiz et al., "Characterization of SpPol4, a unique X-family DNA polymerase in Schizosaccharomyces pombe," Nucleic Acids Research, vol. 33, no. 15, pp. 4762-4774, 2005.

[63] L. Gellon, D. R. Carson, J. P. Carson, and B. Demple, "Intrinsic 5'-deoxyribose-5-phosphate lyase activity in Saccharomyces cerevisiae Trf4 protein with a possible role in base excision DNA repair," DNA Repair, vol. 7, no. 2, pp. 187-198, 2008.

[64] P. Fortini and E. Dogliotti, "Base damage and single-strand break repair: mechanisms and functional significance of short- and long-patch repair subpathways," DNA Repair, vol. 6, no. 4, pp. 398-409, 2007.

[65] L. Wiederhold, J. B. Leppard, P. Kedar et al., "AP endonucleaseindependent DNA base excision repair in human cells," Molecular Cell, vol. 15, no. 2, pp. 209-220, 2004.

[66] S.-W. Yang, A. B. Burgin Jr., B. N. Huizenga, C. A. Robertson, K. C. Yao, and H. A. Nash, "A eukaryotic enzyme that can disjoin dead-end covalent complexes between DNA and type I topoisomerases," Proceedings of the National Academy of Sciences of the United States of America, vol. 93, no. 21, pp. 11534-11539, 1996.

[67] T. S. Dexheimer, S. Antony, C. Marchand, and Y. Pommier, "Tyrosyl-DNA phosphodiesterase as a target for anticancer therapy," Anti-Cancer Agents in Medicinal Chemistry, vol. 8, no. 4, pp. 381-389, 2008.

[68] A. Jilani and D. Ramotar, "Purification and partial characterization of a DNA $3^{\prime}$-phosphatase from Schizosaccharomyces pombe," Biochemistry, vol. 41, no. 24, pp. 7688-7694, 2002.

[69] M. Meijer, F. Karimi-Busheri, T. Y. Huang, M. Weinfeld, and D. Young, "Pnk1, a DNA kinase/phosphatase required for normal response to DNA damage by $\gamma$-radiation or camptothecin in Schizosaccharomyces pombe," The Journal of Biological Chemistry, vol. 277, no. 6, pp. 4050-4055, 2002.

[70] K. V. Inamdar, J. J. Pouliot, T. Zhou, S. P. Lees-Miller, A. Rasouli-Nia, and L. F. Povirk, "Conversion of phosphoglycolate to phosphate termini on $3^{\prime}$ overhangs of DNA double strand breaks by the human tyrosylDNA phosphodiesterase hTdp1," The Journal of Biological Chemistry, vol. 277, no. 30, pp. 27162-27168, 2002.

[71] H. Interthal, H. J. Chen, and J. J. Champoux, "Human Tdp1 cleaves a broad spectrum of substrates, including phosphoamide linkages," The Journal of Biological Chemistry, vol. 280, no. 43, pp. 36518-36528, 2005.

[72] H. Takashima, C. F. Boerkoel, J. John et al., "Mutation of TDP1, encoding a topoisomerase I-dependent DNA damage repair enzyme, in spinocerebellar ataxia with axonal neuropathy," Nature Genetics, vol. 32, no. 2, pp. 267-272, 2002.
[73] S. Ben Hassine and B. Arcangioli, "Tdp1 protects against oxidative DNA damage in non-dividing fission yeast," EMBO Journal, vol. 28, no. 6, pp. 632-640, 2009.

[74] N. Mutoh, C. W. Nakagawa, and K. Yamada, "The role of catalase in hydrogen peroxide resistance in fission yeast Schizosaccharomyces pombe," Canadian Journal of Microbiology, vol. 45, no. 2, pp. 125-129, 1999.

[75] Y. Hida and S. Ikeda, "Base excision repair of oxidative DNA damage in a catalase-deficient mutant of Schizosaccharomyces pombe," Genes and Environment, vol. 30, no. 3, pp. 86-91, 2008.

[76] J. Fan and D. M. Wilson III, "Protein-protein interactions and posttranslational modifications in mammalian base excision repair," Free Radical Biology and Medicine, vol. 38, no. 9, pp. 1121-1138, 2005.

[77] S. S. Y. Su, Y. Tanaka, I. Samejima, K. Tanaka, and M. Yanagida, "A nitrogen starvation-induced dormant $\mathrm{G}_{0}$ state in fission yeast: the establishment from uncommitted $G_{1}$ state and its delay for return to proliferation," Journal of Cell Science, vol. 109, no. 6, pp. 1347-1357, 1996.

[78] S. Mochida and M. Yanagida, "Distinct modes of DNA damage response in $S$. pombe $\mathrm{G}_{0}$ and vegetative cells," Genes to Cells, vol. 11, no. 1, pp. 13-27, 2006. 

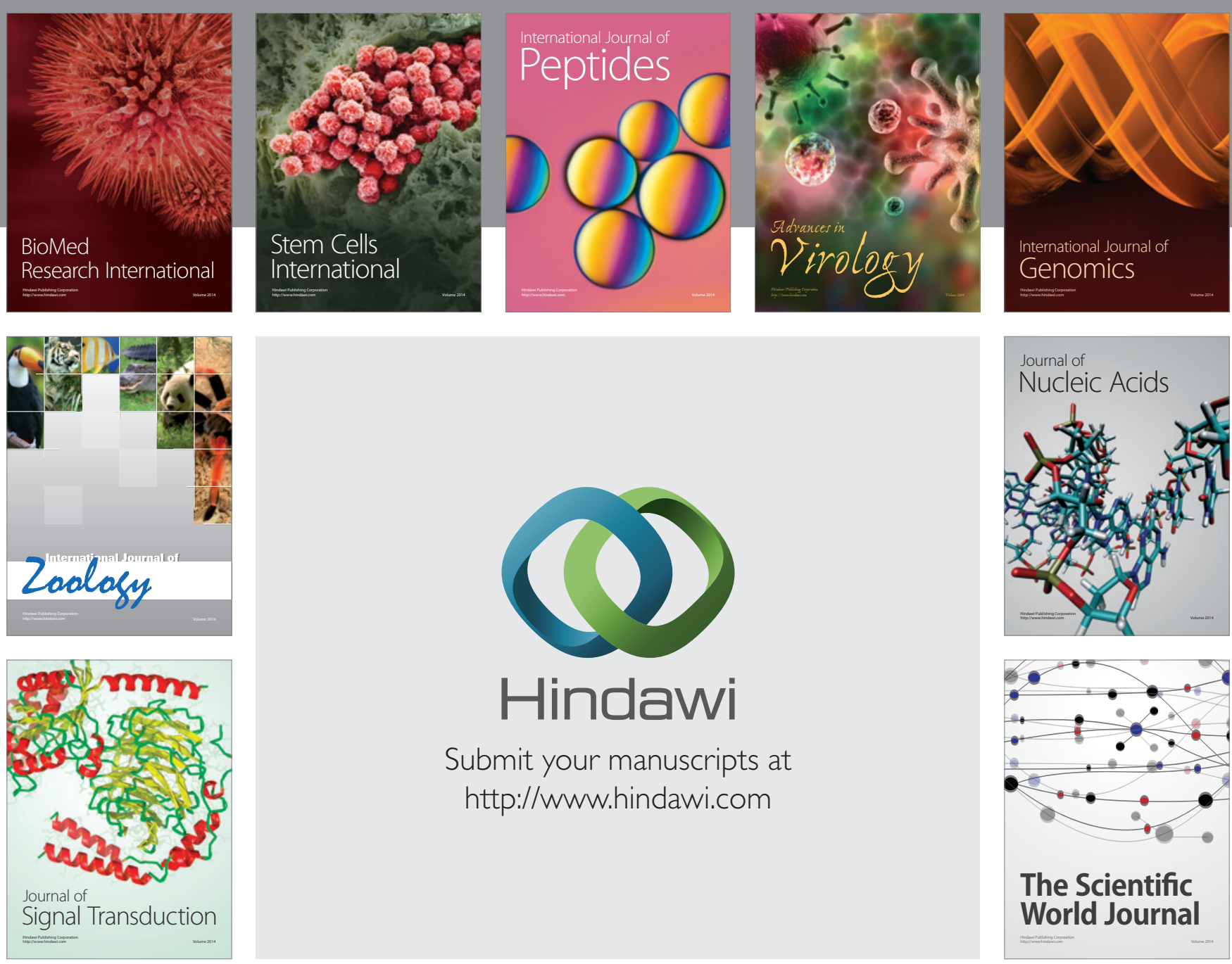

Submit your manuscripts at

http://www.hindawi.com
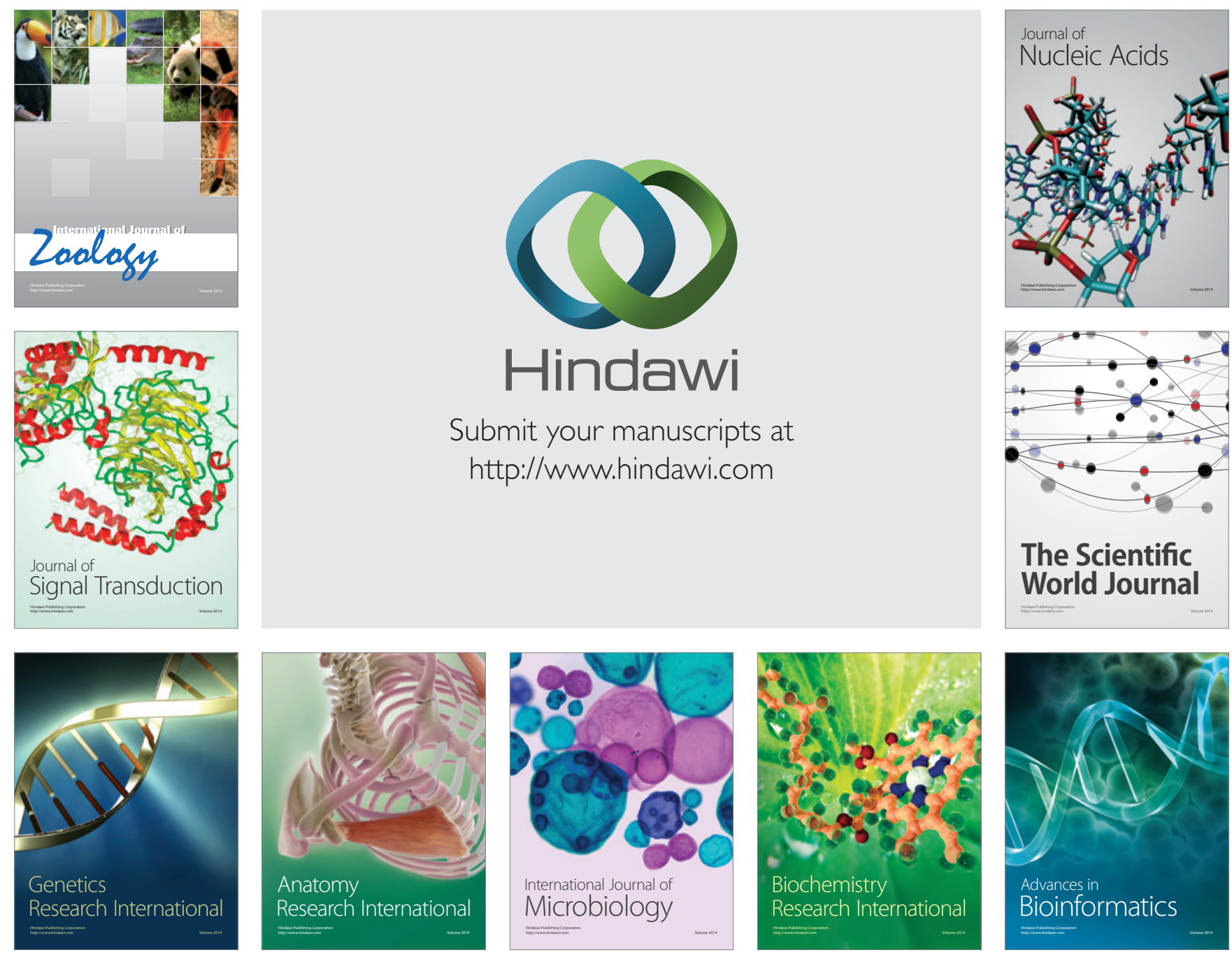

The Scientific World Journal
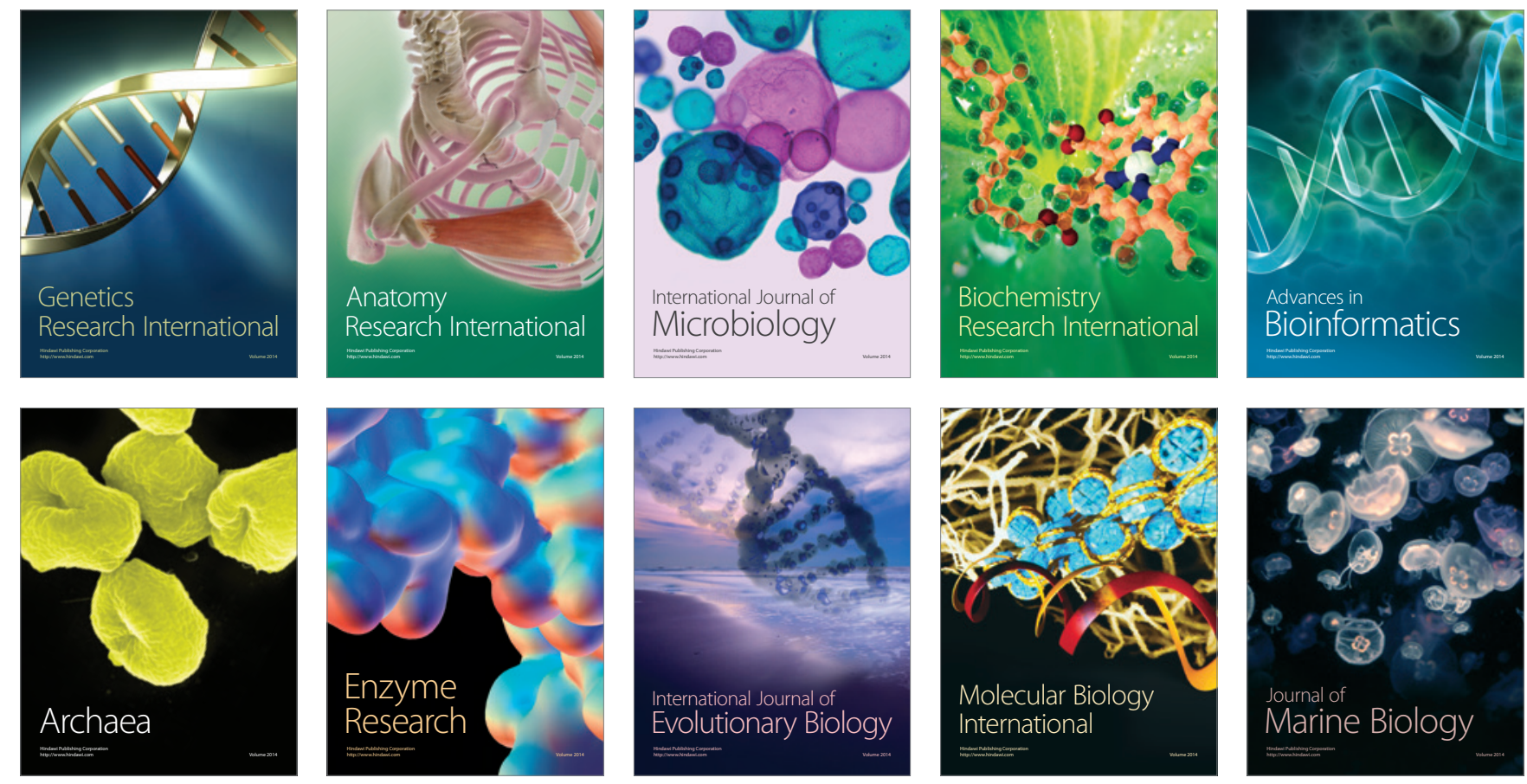